\title{
Interactive Effect of Different Nitrogen and Potash Levels on the Incidence of Bacterial Leaf Blight of Rice (Oryza sativa L.)
}

\author{
Nazer Manzoor, Nadeem Akbar*, Shakeel Ahmad Anjum, Iftikhar Ali, Muhammad Shahid, \\ Abdul Shakoor, Muhammad Waseem Abbas, Khyzer Hayat, Waqas Hamid, M. Asim Rashid \\ Department of Agronomy, University of Agriculture, Faisalabad, Pakistan \\ Email: *bioworld2020@gmail.com
}

How to cite this paper: Manzoor, N., Akbar, N., Anjum, S.A., Ali, I., Shahid, M., Shakoor, A., Abbas, M.W., Hayat, K., Hamid, W. and Rashid, M.A. (2017) Interactive Effect of Different Nitrogen and Potash Levels on the Incidence of Bacterial Leaf Blight of Rice (Oryza sativa L.). Agricultural Sciences, 8, 56-63.

http://dx.doi.org/10.4236/as.2017.81005

Received: December 1, 2016

Accepted: January 16, 2017

Published: January 19, 2017

Copyright $\odot 2017$ by authors and Scientific Research Publishing Inc. This work is licensed under the Creative Commons Attribution International License (CC BY 4.0).

http://creativecommons.org/licenses/by/4.0/

\begin{abstract}
An experiment was conducted at Rice Research Institute, Kala Shah Kaku in 2010 during kharif season to study the influence of nitrogen $(\mathrm{N})$ and potash (K) on severity of Bacterial leaf blight (BLB) of rice (Oryza sativa L.) aimed at improving productivity. The experiment was laid out in randomized complete block design with factorial arrangement with three replications. Treatments comprised of: $0 \mathrm{~kg} \mathrm{~N} \mathrm{ha}{ }^{-1}, 75 \mathrm{~kg} \mathrm{~N} \mathrm{ha}^{-1}, 100 \mathrm{~kg} \mathrm{~N} \mathrm{ha}^{-1}, 125 \mathrm{~kg} \mathrm{~N} \mathrm{ha}^{-1}$ and $0 \mathrm{~kg}$ $\mathrm{K} \mathrm{ha}^{-1}, 50 \mathrm{~kg} \mathrm{~K} \mathrm{ha}^{-1}, 75 \mathrm{~kg} \mathrm{~K} \mathrm{ha}^{-1}, 100 \mathrm{~kg} \mathrm{~K} \mathrm{ha}^{-1}$. Data on disease severity and paddy yield were recorded using standard procedures. Paddy yield was affected significantly by various combinations of N \& K. In case of bacterial leaf blight, minimum diseased incidence percentage was observed when nitrogen alone was applied @ $75 \mathrm{~kg} \cdot h a^{-1}$ in contrast to fertilizer applied @ $125 \mathrm{~kg} \mathrm{~N} \mathrm{ha}^{-1}$ $+50 \mathrm{~kg} \mathrm{~K} \mathrm{ha}{ }^{-1}$ which showed maximum diseased incidence percentage. Maximum paddy yield (4.32 t.ha $\left.{ }^{-1}\right)$ was recorded when rice was fertilized @ 75 $\mathrm{kg} \mathrm{N} \mathrm{ha}^{-1}+100 \mathrm{~kg} \mathrm{~K} \mathrm{ha}^{-1}$ as compared to sole fertilization of $75 \mathrm{~kg} \mathrm{~K} \mathrm{ha}^{-1}$ that produced minimum paddy yield $\left(2.40 \mathrm{t} \cdot \mathrm{ha}^{-1}\right)$. Maximum gross income, net returns and benefit cost ratio were obtained where rice crop was fertilized @ $75 \mathrm{~kg} \mathrm{~N} \mathrm{ha}^{-1}$ and $100 \mathrm{~kg} \mathrm{~K} \mathrm{ha}^{-1}$.
\end{abstract}

\section{Keywords}

Rice, Bacterial Leaf Blight, Paddy Yield, Harvest Index, Benefit Cost Ratio

\section{Introduction}

Rice (Oryza sativa L.) is an important cereal crop and nearly more than half population of the world subsists on it. It is the third largest crop in Pakistan after wheat and cotton [1]. Despite higher potential, average yield of rice in Pakistan 
is $2.38 \mathrm{t} \cdot \mathrm{ha}^{-1}$ which is lower than most countries in the world. This low production is attributed to delayed sowing, low plant population, imbalance fertilizer use and disease attack especially Rice blast and Bacterial leaf blight [2]. Rice blast and BLB of rice are more widespread in Pakistan [3]. The bacterial leaf blight disease is reported to induce $50 \%$ or even greater losses in severe cases. Losses in the Tropical Asia varied 2\% - 74\% depending upon location, weather conditions, crop stage and cultivar [4].

Effective control of the disease had not been achieved by chemical methods [5]. Under prevailing conditions commercial resistant varieties are generally scarce and current varieties are susceptible to these diseases thus reducing their potential due to disease attack. Cultural practices such as fertilizer rate, sowing time and irrigation etc. played an important role for the control of these diseases [6]. So, there is a need to emphasize over the cultural techniques for the effective management of these diseases. The plant diseases are influenced by specific genotype, fertilizer input and climatic conditions, which vary yearly [7]. Different plant nutrients and their balanced use played a significant role in reducing pest and disease infestation, which results in higher returns through enhanced yields and better quality [8]. The cultural control may include the alteration of nitrogen $(\mathrm{N})$ and potash $(\mathrm{K})$ levels and investigating the appropriate doses for the control of these diseases. Optimum fertilization of $\mathrm{N}$ and $\mathrm{K}$ induces disease tolerance in plants.

It is a fact that excessive $\mathrm{N}$ application predisposes plants to disease caused by obligatory parasitic pathogens [9]. Excessive use of $\mathrm{N}$ fertilizers could result in more leaf growth that was over succulent and more susceptible to certain diseases [10]. It was found that BLB incidence considerably exaggerated with increasing rate of $\mathrm{N}$ fertilizer [11]. On the other hand, it was found that low $\mathrm{N}$ fertilizer use increased susceptibility to disease attack. BLB was considerably more severe on the vulnerable cultivars when $\mathrm{N}$ fertilizer was added in low rates. Nitrogen deficiency induced modification of many morphological and physiological parameters, limitation of growth, leaf number and leaf area [12]. It found that narrow brown leaf spot was more severe in low $\mathrm{N}$ treatments [13] [14] [15]; they also emphasized on the positive role of $\mathrm{N}$ fertilization for minimizing disease incidence [7].

The role of $\mathrm{K}$ is very important regarding the disease control. The potassium aggregate increases the vigor and the resistance of the plant to stress. It fortifies the straw, enhances the quality of grains and helps the transfer of starch, sugars and oils. $\mathrm{K}$ strengthens the plant leaf cells and $\mathrm{K}$ deficiency in leaf cells makes them weak and susceptible to secondary fungal infection [16]. Sufficient K nutrition increases cell cuticle thickness, cell wall strength and production of phenols that ultimately implant resistance in crops [17]. Potassium fertilization greatly affected panicle blast development, the response being significantly linear and negative with increasing levels of potash [18]. Thus, disease incidence can be lowered by high K levels [19] [20]. However, in Pakistan K use is only about 2 $\mathrm{kg} \cdot \mathrm{ha}^{-1}[1]$. 
Contrarily, [21] found that $\mathrm{K}$ did not affect the disease severity of Anthracnose crown rot (Colletotrichum fragariae) on strawberry plants. $\mathrm{K}$ fertilization did not have a noticeable effect on disease severity scores. Similarly, [13] investigated the effect of $\mathrm{K}$ fertilization on the severity of Narrow brown leaf spot in rice. The present study was therefore planned to evaluate the influence of $\mathrm{N}$ and $\mathrm{K}$ solely or in combination, on the incidence of BLB of rice.

\section{Materials and Methods}

A research to study influence of $\mathrm{N}$ and $\mathrm{K}$ levels on the severity of BLB was conducted at Rice Research Institute (RRI), Kala Shah Kaku, Lahore, during kharif season, 2010. The experiment was laid out in a Randomized Complete Block Design (RCBD) with factorial arrangement comprising three replications using net plot size of $2.25 \mathrm{~m} \times 6.0 \mathrm{~m}$. The treatments of an experiment comprised; $0 \mathrm{~kg} \mathrm{~N}$ ha $^{-1}$ (control), $75 \mathrm{~kg} \mathrm{~N} \mathrm{ha}^{-1}, 100 \mathrm{~kg} \mathrm{~N} \mathrm{ha}^{-1}, 125 \mathrm{~kg} \mathrm{~N} \mathrm{ha}^{-1}$ and $0 \mathrm{~kg} \mathrm{~K} \mathrm{ha}^{-1}$ (control), $50 \mathrm{~kg} \mathrm{~K} \mathrm{ha}^{-1}, 75 \mathrm{~kg} \mathrm{~K} \mathrm{ha}^{-1}, 100 \mathrm{~kg} \mathrm{~K} \mathrm{ha}^{-1}$. Thirty days old nursery of Super Basmati was transplanted manually in puddled field on $16^{\text {th }}$ July, 2010. Phosphorous fertilizer was added @ $75 \mathrm{~kg} \cdot \mathrm{ha}^{-1}$. Whole of the phosphorus and zinc was applied at the time of soil preparation. Full dose of $\mathrm{K}$ were added to the plots just before transplanting of rice nursery according to respective treatments. $\mathrm{N}$ was added in two splits i.e. $1 / 2$ of $\mathrm{N}$ was applied just after transplantation of nursery and remaining $1 / 2$ half dose was applied 30 days after transplanting (DAT). Machete 60EC was applied @ $2 \mathrm{t} /$ ha to control weeds just after four days of transplanting. The remaining (fallow) weeds were controlled manually. $\mathrm{ZnSO}_{4}$ (35\%) was applied @ $12.5 \mathrm{~kg} \cdot \mathrm{ha}^{-1}$ at 12 DAT. Rice was inoculated with Xanthomonas oryzae pv. oryzae following clipping method at tillering and heading stage. Pathogen was inoculated by piercing method. The leaves were inoculated at booting stage. Paddy yield was recorded after harvesting each plot and was converted into th $\mathrm{ha}^{-1}$. Data regarding BLB incidence was analyzed following disease rating scales (Table 1) developed by IRRI [22]. Percent disease incidence was calculated according to the formula as follows:

Disease Incidence $(\%)=($ Total lesion area $) /($ Total leaf length $) \times 100$

\section{Statistical Analysis}

The data obtained were analyzed statistically by using Fisher analysis of variance technique and difference among treatment means was compared by using least

Table 1. Disease rating scale for assessment of BLB in rice crop.

\begin{tabular}{ccc}
\hline Disease incidence (\%) & Response & Ranking \\
\hline $0 \%$ & Highly resistant & 0 \\
$0 \%-1 \%$ & Resistant & 1 \\
$1 \%-5 \%$ & Moderately resistant & 3 \\
$6 \%-25 \%$ & Moderately susceptible & 5 \\
$26 \%-50 \%$ & Susceptible & 7 \\
$51 \%-100 \%$ & Highly susceptible & 9 \\
\hline
\end{tabular}


significant difference test (LSD) at 5\% probability level. The differences were only considered when significant at $\mathrm{p}<0.05$ [23].

\section{Results and Discussion}

The analysis of variance confirmed the statistically significant variation among the different rates of nitrogen $(\mathrm{N})$ application as well as among the different rates of potassium (K) application. Moreover, interactive effects of $\mathrm{N}$ and $\mathrm{K}$ application were also found to be statistically significant.

The results showed that disease incidence of bacterial leaf blight (BLB) in rice decreased as $\mathrm{K}$ application increased at $0 \mathrm{~kg} \mathrm{ha}^{-1} \mathrm{~N}$ but this decreased was not statistically significant over control (no application of $\mathrm{N}$ and $\mathrm{K}$ ). However, at 75 $\mathrm{kg} \mathrm{ha}^{-1} \mathrm{~N}, \mathrm{~K}$ application decreased the disease incidence significantly. The minimum disease incidence of BLB (15.76\%) in rice was observed when $75 \mathrm{~kg} \mathrm{ha}^{-1} \mathrm{~N}$ was applied with no application of $\mathrm{K}$ and these results were followed by the application of $75 \mathrm{~kg} \mathrm{ha}^{-1} \mathrm{~N}$ with $100 \mathrm{~kg} \mathrm{ha}^{-1} \mathrm{~K}$ where disease incidence was $21.22 \%$. However, application of $75 \mathrm{~kg} \mathrm{ha}^{-1} \mathrm{~N}$ with $0 \mathrm{~kg} \mathrm{ha}^{-1} \mathrm{~K}$ was statistically at par with the application of $75 \mathrm{~kg} \mathrm{ha}^{-1} \mathrm{~N}$ with $100 \mathrm{~kg} \mathrm{ha}^{-1} \mathrm{~K}$ but statistically significant from all other combinations of $\mathrm{N}$ and $\mathrm{K}$ in terms of disease incidence in rice. Maximum disease incidence in rice was observed when $125 \mathrm{~kg} \mathrm{ha}^{-1} \mathrm{~N}$ was applied with $50 \mathrm{~kg} \mathrm{ha}^{-1} \mathrm{~K}$ followed by control (no application of $\mathrm{N}$ and $\mathrm{K}$ ) (Table 2). The percent diseased leaf area showed a parabolic trend to various $\mathrm{N}$ fertilization levels at $0 \mathrm{~kg} \mathrm{~K} \mathrm{ha}^{-1}$ i.e. more where no $\mathrm{N}$ was applied, decreased by $55 \%$ when $\mathrm{N}$ application was made at the rate of $75 \mathrm{~kg} \mathrm{~N} \mathrm{ha}^{-1}$, and again increased by $11.9 \%$ to $26.6 \%$ at $100 \mathrm{~kg} \mathrm{~N} \mathrm{ha}^{-1}$ to $125 \mathrm{~kg} \mathrm{~N} \mathrm{ha}^{-1}$ against control ( $0 \mathrm{~kg}$ $\mathrm{N} \mathrm{ha}^{-1}$ ). At $50 \mathrm{~kg} \mathrm{~K} \mathrm{ha}^{-1}$ level, the percent diseased leaf area remained unaffected with $\mathrm{N}$ fertilization from $0 \mathrm{~kg} \mathrm{~N} \mathrm{ha}^{-1}$ to $100 \mathrm{~kg} \mathrm{~N} \mathrm{ha}^{-1}$, however percent diseased leaf area (\% DLA) reaches at its maximum at $125 \mathrm{~kg} \mathrm{~N} / \mathrm{ha}$. On the other hand, percent diseased leaf area (\% DLA) remained unaffected with any rate of $\mathrm{N}$ at 75 $\mathrm{kg} \mathrm{K} \mathrm{ha}{ }^{-1}$ level. The percent diseased leaf area (\% DLA) once again showed a parabolic trend at $100 \mathrm{~kg} \mathrm{~K}$ level where comparatively high percent diseased leaf area (\% DLA) was recorded in treatment without $\mathrm{N}$ fertilization and it decreased by $24.48 \%$ at $75 \mathrm{~kg} \mathrm{~N} \mathrm{ha}^{-1}$ level. A further increase of $\mathrm{N}$ fertilization by $25 \mathrm{~kg} \mathrm{~N}$ $\mathrm{ha}^{-1}$ (i.e. $100 \mathrm{~kg} \mathrm{~N} \mathrm{ha}^{-1}$ ) did not contributed any way to percent diseased leaf area (\% DLA). However, when $\mathrm{N}$ fertilization was increased up to $125 \mathrm{~kg} \mathrm{~N} \mathrm{ha}^{-1}$ the percent diseased leaf area (\% DLA) again increased by $33 \%$. Percent diseased leaf area (\% DLA) varied differently in relation to $\mathrm{N}$ fertilization at different $\mathrm{K}$ rates. Irregular trend was seen at different $\mathrm{K}$ levels however, $75 \mathrm{~kg} \mathrm{~N} \mathrm{ha}^{-1}$ to $100 \mathrm{~kg} \mathrm{~N}$ $\mathrm{ha}^{-1}$ gave lower percent diseased leaf area (\% DLA) as compared to control and $125 \mathrm{~kg} \mathrm{~N} \mathrm{ha}^{-1}$. Based on results, it was deduced that BLB could be minimized by a better combination of $\mathrm{N}$ and $\mathrm{K}$ rather to apply these nutrients in a haphazard manner. So for better management of disease a balanced combination of $\mathrm{N}$ and $\mathrm{K}$ should be applied. It is suggested that $\mathrm{K}: \mathrm{N}$ ratio is more important than the effect of each individual nutrient in blast development [18].

At no $\mathrm{N}$ application, non-significant increase in paddy yield was observed by 
Table 2. Effect of nitrogen and potassium on the diseases incidence of bacterial leaf blight and paddy yield of rice.

\begin{tabular}{|c|c|c|c|}
\hline \multicolumn{2}{|c|}{ Treatments } & \multirow{2}{*}{$\begin{array}{c}\text { Disease Incidence of } \\
\text { BLB (\%) }\end{array}$} & \multirow{2}{*}{$\begin{array}{c}\text { Paddy Yield }\left(\mathrm{kg} \cdot \mathrm{ha}^{-1}\right) \\
2.46 \mathrm{~d}\end{array}$} \\
\hline \multirow{5}{*}{ 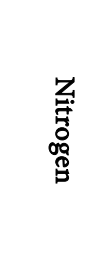 } & $0 \mathrm{~kg} \cdot \mathrm{ha}^{-1}$ & & \\
\hline & $75 \mathrm{~kg} \cdot \mathrm{ha}^{-1}$ & $21.05 \mathrm{~d}$ & $3.98 \mathrm{a}$ \\
\hline & $100 \mathrm{~kg} \cdot \mathrm{ha}^{-1}$ & $25.59 \mathrm{c}$ & $3.78 \mathrm{~b}$ \\
\hline & $125 \mathrm{~kg} \cdot \mathrm{ha}^{-1}$ & $33.74 \mathrm{a}$ & $3.30 \mathrm{c}$ \\
\hline & LSD value & 3.41 & 0.14 \\
\hline \multirow{5}{*}{ 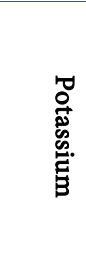 } & $0 \mathrm{~kg} \cdot \mathrm{ha}^{-1}$ & $26.99 \mathrm{~b}$ & $3.22 \mathrm{c}$ \\
\hline & $50 \mathrm{~kg} \cdot \mathrm{ha}^{-1}$ & $31.33 \mathrm{a}$ & $3.36 \mathrm{bc}$ \\
\hline & $75 \mathrm{~kg} \cdot \mathrm{ha}^{-1}$ & $25.89 \mathrm{~b}$ & $3.40 \mathrm{ab}$ \\
\hline & $100 \mathrm{~kg} \cdot \mathrm{ha}^{-1}$ & $26.35 \mathrm{~b}$ & $3.53 \mathrm{a}$ \\
\hline & LSD value & 3.41 & 0.14 \\
\hline \multirow{17}{*}{ 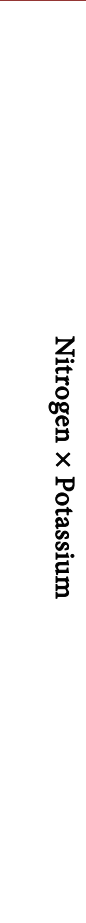 } & $0 \mathrm{~kg} \mathrm{~N} \mathrm{ha}^{-1} \times 0 \mathrm{~kg} \mathrm{~K} \mathrm{ha}^{-1}$ & $35.27 \mathrm{~b}$ & $2.44 \mathrm{f}$ \\
\hline & $0 \mathrm{~kg} \mathrm{~N} \mathrm{ha}^{-1} \times 50 \mathrm{~kg} \mathrm{Kha}^{-1}$ & $30.20 \mathrm{bcde}$ & $2.47 \mathrm{f}$ \\
\hline & $0 \mathrm{~kg} \mathrm{~N} \mathrm{ha}^{-1} \times 75 \mathrm{~kg} \mathrm{Kha}^{-1}$ & $27.17 \mathrm{cdefg}$ & $2.40 \mathrm{f}$ \\
\hline & $0 \mathrm{~kg} \mathrm{~N} \mathrm{ha}^{-1} \times 100 \mathrm{~kg} \mathrm{~K} \mathrm{ha}^{-1}$ & $28.10 \mathrm{cdef}$ & $2.51 \mathrm{f}$ \\
\hline & $75 \mathrm{~kg} \mathrm{~N} \mathrm{ha}^{-1} \times 0 \mathrm{~K} \mathrm{~kg} \mathrm{ha}^{-1}$ & $15.76 \mathrm{~h}$ & $3.62 \mathrm{~b}$ \\
\hline & $75 \mathrm{~kg} \mathrm{~N} \mathrm{ha}^{-1} \times 50 \mathrm{~K} \mathrm{~kg} \mathrm{ha}^{-1}$ & $24.13 \mathrm{efg}$ & $3.89 \mathrm{~b}$ \\
\hline & $75 \mathrm{~kg} \mathrm{~N} \mathrm{ha}^{-1} \times 75 \mathrm{~K} \mathrm{~kg} \mathrm{ha}^{-1}$ & $23.07 \mathrm{fg}$ & $4.09 \mathrm{a}$ \\
\hline & $75 \mathrm{~kg} \mathrm{~N} \mathrm{ha}^{-1} \times 100 \mathrm{~K} \mathrm{~kg} \mathrm{ha}^{-1}$ & $21.22 \mathrm{gh}$ & $4.32 \mathrm{a}$ \\
\hline & $100 \mathrm{~kg} \mathrm{~N} \mathrm{ha}^{-1} \times 0 \mathrm{~kg} \mathrm{~K} \mathrm{ha}^{-1}$ & $25.89 \mathrm{defg}$ & $3.68 \mathrm{bc}$ \\
\hline & $100 \mathrm{~kg} \mathrm{~N} \mathrm{ha}^{-1} \times 50 \mathrm{~kg} \mathrm{~K} \mathrm{ha}^{-1}$ & 27.82 cdefg & $3.83 \mathrm{~b}$ \\
\hline & $100 \mathrm{~kg} \mathrm{~N} \mathrm{ha}^{-1} \times 75 \mathrm{~kg} \mathrm{~K} \mathrm{ha}^{-1}$ & $26.33 \mathrm{defg}$ & $3.79 \mathrm{~b}$ \\
\hline & $100 \mathrm{~kg} \mathrm{~N} \mathrm{ha}^{-1} \times 100 \mathrm{~kg} \mathrm{~K} \mathrm{ha}^{-1}$ & $22.33 \mathrm{f}$ & $3.80 \mathrm{~b}$ \\
\hline & $125 \mathrm{~N} \mathrm{~kg} \mathrm{ha}^{-1} \times 0 \mathrm{~kg} \mathrm{~K} \mathrm{ha}^{-1}$ & $31.06 \mathrm{bcd}$ & $3.14 \mathrm{e}$ \\
\hline & $125 \mathrm{~N} \mathrm{~kg} \mathrm{ha}^{-1} \times 50 \mathrm{~kg} \mathrm{~K} \mathrm{ha}^{-1}$ & $43.17 \mathrm{a}$ & $3.25 \mathrm{de}$ \\
\hline & $125 \mathrm{~N} \mathrm{~kg} \mathrm{ha}^{-1} \times 75 \mathrm{~kg} \mathrm{~K} \mathrm{ha}^{-1}$ & $27.00 \mathrm{cdefg}$ & $3.34 \mathrm{de}$ \\
\hline & $125 \mathrm{~N} \mathrm{~kg} \mathrm{ha}^{-1} \times 100 \mathrm{~kg} \mathrm{~K} \mathrm{ha}^{-1}$ & $33.73 \mathrm{bc}$ & $3.48 \mathrm{~cd}$ \\
\hline & LSD value & 6.837 & 0.28 \\
\hline
\end{tabular}

Note: Values sharing the different letters differ significantly at 0.05 probability; NS = Non-Significant.

$\mathrm{K}$ application. However, at $75 \mathrm{~kg} \mathrm{~N} \mathrm{ha}{ }^{-1}, \mathrm{~K}$ application increased paddy yield significantly. Further increase in $\mathrm{N}$ dose along with $\mathrm{K}$ didn't influence paddy yield significantly. Maximum paddy yield $\left(4.32 \mathrm{t} \cdot \mathrm{ha}^{-1}\right)$ was recorded with the application of $75 \mathrm{~kg} \mathrm{ha}^{-1} \mathrm{~N}$ with $100 \mathrm{~kg} \mathrm{ha}^{-1} \mathrm{~K}$ followed by $75 \mathrm{~kg} \mathrm{ha}^{-1} \mathrm{~N}$ with $75 \mathrm{~kg}$ $\mathrm{ha}^{-1} \mathrm{~K}$. Application of $75 \mathrm{~kg} \mathrm{ha}^{-1} \mathrm{~N}$ with $100 \mathrm{~kg} \mathrm{ha}^{-1} \mathrm{~K}$ was statistically at par with the application of $75 \mathrm{~kg} \mathrm{ha}^{-1} \mathrm{~N}$ with $75 \mathrm{~kg} \mathrm{ha}^{-1} \mathrm{~K}$ but statistically significant from all other combinations of $\mathrm{N}$ and $\mathrm{K}$ in terms of paddy yield. Minimum paddy yield was recorded in control (no application of $\mathrm{N}$ and $\mathrm{K}$ ). $\mathrm{K}$ application increased the paddy yield but this increase was statistically not significant at $0 \mathrm{~kg}$ $\mathrm{ha}^{-1} \mathrm{~N}$ (Table 2). Results indicated a marked increase in yield from $0 \mathrm{~kg} \mathrm{~N} \mathrm{ha}^{-1}$ 
to $100 \mathrm{~kg} \mathrm{~N} \mathrm{ha}^{-1}$. This increase in yield was attributed to more number of panicle bearing tillers, more 1000 kernel weight and more number of kernels per panicle at $75 \mathrm{~kg} \mathrm{~N} \mathrm{ha}^{-1}$ and $100 \mathrm{~kg} \mathrm{~N} \mathrm{ha}^{-1}$. This increase in yield was supported by [15]; they found an increase in yield with increased $\mathrm{N}$ rates. An increase in yield with increasing $\mathrm{N}$ fertilization was observed. However, there was a significant decrease in yield at $125 \mathrm{~kg} \mathrm{~N} \mathrm{ha}^{-1}$ irrespective of different $\mathrm{K}$ levels. This decrease in yield may be due to high disease percentage [6] [19]. Similarly, an increase in yield up to $85 \mathrm{~kg} \mathrm{~N} \mathrm{ha}^{-1}$ was observed beyond this level decrease in yield was attributed to accumulative effect of $\mathrm{N}$ toxicity and disease severity. The yield also displayed a positive increase with increase in $\mathrm{K}$ fertilization [2]. These results were advocated by [17] they explained that potassium fertilization increased the yield over the control.

Economic analysis revealed that application of $75 \mathrm{~kg} \mathrm{~N} \mathrm{ha}^{-1}$ with $100 \mathrm{~kg} \mathrm{~K} \mathrm{ha}^{-1}$ gave maximum gross income of Rs. 154,652 ha $\mathrm{ha}^{-1}$ (Table 3). The highest gross income at this level is attributed to highest yield at the fertilization rate. Application of $75 \mathrm{~kg} \mathrm{~N} \mathrm{ha}^{-1}$ with $100 \mathrm{~kg} \mathrm{~K} \mathrm{ha}^{-1}$ gave maximum net returns amounting Rs. 37159. The highest cost benefit ratio of 1.32 was obtained by $75 \mathrm{~kg} \mathrm{~N} \mathrm{ha}^{-1}$ with $100 \mathrm{~kg} \mathrm{~K} \mathrm{ha}^{-1}$ and $100 \mathrm{~kg} \mathrm{~N} \mathrm{ha}^{-1}$ and $0 \mathrm{~kg} \mathrm{~K} \mathrm{ha}^{-1}$. The production level at $\mathrm{T}_{8}$ is favored for lower disease severity.

Table 3. Economic analysis of different application rates of $\mathrm{N}$ and $\mathrm{K}$ in rice.

\begin{tabular}{|c|c|c|c|c|c|c|c|c|c|}
\hline Treatments & $\begin{array}{c}\text { Yield } \\
\left(\mathrm{t} \cdot \mathrm{ha}^{-1}\right)\end{array}$ & $\begin{array}{c}\text { Paddy } \\
\text { income } \\
\left(\text { Rs. ha }{ }^{-1}\right)\end{array}$ & $\begin{array}{c}\text { Straw } \\
\text { yield } \\
\left(\mathrm{t} \cdot \mathrm{ha}^{-1}\right)\end{array}$ & $\begin{array}{c}\text { Straw } \\
\text { income } \\
\left(\text { Rs. ha }{ }^{-1}\right)\end{array}$ & $\begin{array}{c}\text { Gross } \\
\text { income } \\
\left(\text { Rs. ha }^{-1}\right)\end{array}$ & $\begin{array}{c}\text { Variable } \\
\text { cost } \\
\left(\text { Rs. ha }{ }^{-1}\right)\end{array}$ & $\begin{array}{l}\text { Total cost } \\
\left(\text { Rs. ha }{ }^{-1}\right)\end{array}$ & $\begin{array}{c}\text { Net } \\
\text { returns } \\
\left(\text { Rs. ha }{ }^{-1}\right)\end{array}$ & BCR \\
\hline $0 \mathrm{~kg} \mathrm{ha}^{-1} \mathrm{~N} \times 0 \mathrm{~kg} \mathrm{ha}^{-1} \mathrm{~K}$ & 2.44 & 79,408 & 13.0 & 11,011 & 90,420 & 7941 & 93,252 & -2832 & 0.97 \\
\hline $0 \mathrm{~kg} \mathrm{ha}^{-1} \mathrm{~N} \times 50 \mathrm{~kg} \mathrm{ha}^{-1} \mathrm{~K}$ & 2.47 & 80,167 & 13.1 & 11,138 & 91,305 & 15,667 & 100,978 & -9673 & 0.90 \\
\hline $0 \mathrm{~kg} \mathrm{ha}^{-1} \mathrm{~N} \times 75 \mathrm{~kg} \mathrm{ha}^{-1} \mathrm{~K}$ & 2.40 & 78,000 & 12.9 & 10,956 & 88,956 & 19,275 & 104,586 & $-15,630$ & 0.85 \\
\hline $0 \mathrm{~kg} \mathrm{ha}^{-1} \mathrm{~N} \times 100 \mathrm{~kg} \mathrm{ha}^{-1} \mathrm{~K}$ & 2.51 & 81,683 & 13.6 & 11,530 & 93,214 & 23,468 & 108,779 & $-15,566$ & 0.86 \\
\hline $75 \mathrm{~kg} \mathrm{ha}^{-1} \mathrm{~N} \times 0 \mathrm{~kg} \mathrm{ha}^{-1} \mathrm{~K}$ & 3.62 & 117,758 & 16.8 & 14,312 & 132,071 & 14,628 & 99,939 & 32,131 & 1.32 \\
\hline $75 \mathrm{~kg} \mathrm{ha}^{-1} \mathrm{~N} \times 50 \mathrm{~kg} \mathrm{ha}^{-1} \mathrm{~K}$ & 3.89 & 126,533 & 17.2 & 14,643 & 141,176 & 23,156 & 108,467 & 32,709 & 1.30 \\
\hline $75 \mathrm{~kg} \mathrm{ha}^{-1} \mathrm{~N} \times 75 \mathrm{~kg} \mathrm{ha}^{-1} \mathrm{~K}$ & 4.09 & 132,925 & 16.8 & 14,287 & 147,212 & 27,620 & 112,931 & 34,281 & 1.30 \\
\hline $75 \mathrm{~kg} \mathrm{ha}^{-1} \mathrm{~N} \times 100 \mathrm{~kg} \mathrm{ha}^{-1} \mathrm{~K}$ & 4.32 & 140,292 & 16.9 & 14,360 & 154,652 & 32,182 & 117,493 & 37,159 & 1.32 \\
\hline $100 \mathrm{~kg} \mathrm{ha}^{-1} \mathrm{~N} \times 0 \mathrm{~kg} \mathrm{ha}^{-1} \mathrm{~K}$ & 3.68 & 119,600 & 16.4 & 13,926 & 133,526 & 15,766 & 101,077 & 32,449 & 1.32 \\
\hline $100 \mathrm{~kg} \mathrm{ha}^{-1} \mathrm{~N} \times 50 \mathrm{~kg} \mathrm{ha}^{-1} \mathrm{~K}$ & 3.83 & 124,583 & 16.7 & 14,226 & 138,810 & 23,915 & 109,226 & 29,584 & 1.27 \\
\hline $100 \mathrm{~kg} \mathrm{ha}^{-1} \mathrm{~N} \times 75 \mathrm{~kg} \mathrm{ha}^{-1} \mathrm{~K}$ & 3.79 & 123,067 & 17.2 & 14,653 & 137,719 & 27,588 & 112,899 & 24,820 & 1.22 \\
\hline $100 \mathrm{~kg} \mathrm{ha}^{-1} \mathrm{~N} \times 100 \mathrm{~kg} \mathrm{ha}^{-1} \mathrm{~K}$ & 3.80 & 123,608 & 16.8 & 14,295 & 137,904 & 31,467 & 116,778 & 21,126 & 1.18 \\
\hline $125 \mathrm{~kg} \mathrm{ha}^{-1} \mathrm{~N} \times 0 \mathrm{~kg} \mathrm{ha}^{-1} \mathrm{~K}$ & 3.14 & 102,158 & 15.9 & 13,528 & 115,686 & 14,967 & 100,278 & 15,408 & 1.15 \\
\hline $125 \mathrm{~kg} \mathrm{ha}^{-1} \mathrm{~N} \times 50 \mathrm{~kg} \mathrm{ha}^{-1} \mathrm{~K}$ & 3.25 & 105,733 & 15.8 & 13,431 & 119,164 & 22,975 & 108,286 & 10,878 & 1.10 \\
\hline $125 \mathrm{~kg} \mathrm{ha}^{-1} \mathrm{~N} \times 75 \mathrm{~kg} \mathrm{ha}^{-1} \mathrm{~K}$ & 3.34 & 108,550 & 16.2 & 13,749 & 122,299 & 27,081 & 112,392 & 9907 & 1.09 \\
\hline $125 \mathrm{~kg} \mathrm{ha}^{-1} \mathrm{~N} \times 100 \mathrm{~kg} \mathrm{ha}^{-1} \mathrm{~K}$ & 3.48 & 113,100 & 16.9 & 14,339 & 127,439 & 31,361 & 116,672 & 10,767 & 1.09 \\
\hline
\end{tabular}

Fixed cost Rs. ha ${ }^{-1}=85,311.0$, Straw price Rs. per ton $=850.0$, Paddy price Rs. per $40 \mathrm{~kg}=1300.0, \mathrm{BCR}=$ Benefit cost ratio, $\mathrm{N}=$ Nitrogen, $\mathrm{K}=$ Potassium. 


\section{Conclusion}

Rice blast responded significantly to $\mathrm{N}$ fertilization. Both deficiency and excessive use of $\mathrm{N}$ promoted disease severity. BLB also responded significantly to $\mathrm{K}$ fertilization. Both deficiency and excessive use of N promoted BLB disease severity. Lower diseased leaf area (\% DLA) was observed where $\mathrm{N}$ was applied at the rate of $75-100 \mathrm{~kg} \cdot \mathrm{ha}^{-1}$. Results revealed that balanced nutrition is required to BLB. As the rate of $\mathrm{N}$ fertilizer was increased the demand for potassium also increased. Super Basmati gave best paddy yield, highest harvest index value, higher gross income, maximum net returns, higher benefit cost ratio and more resistance against BLB when fertilized @ $75 \mathrm{~kg} \mathrm{~N} \mathrm{ha}^{-1} \& 100 \mathrm{~kg} \mathrm{~K} \mathrm{ha}^{-1}$.

\section{References}

[1] Anonymous (2010) Economic Survey of Pakistan 2009-10. Ministry of Food Agriculture and Livestock Division (Economic Wing), Government of Pakistan, Islamabad, 22-23.

[2] Chaudhary, A.U., Hussain, M., Iqbal, J. and Ali, M.A. (2009) Effect of Nitrogen Doses on Incidence of BLB in Rice. Journal of Agricultural Research, 3, 253-257.

[3] Ou, S.H. (1985) Rice Diseases. 2nd Edition, Commonwealth Mycological Institute, Kew, $380 \mathrm{p}$

[4] Davierwala, A.P., Reddy, A.P.K., Lagu, M.D., Ranjekar, P.K. and Gupta, V.S. (2001) Marker Assisteel Selection of Bacterial Blight Resistant Gene in Rice. Biochemical Genetics, 7-8, 261-178. https://doi.org/10.1023/A:1010282732444

[5] Reissing, W.H., Heinrich, E.A., Listinger, I.A. and Barrian, A. (1986) Illustrated Guide to Integrated Pest Management in Rice. Tropical Asia IRRI, Manaila Philippines, $411 \mathrm{p}$.

[6] Myint, S.S., Nyut, H.K., Ko, H.K. and Thein, M.M. (2007) Study on the Effect of Different Urea Fertilizer Rates and Plant Populations on the Severity of Bacterial Blight of Rice. Journal of Agriculture \& Development in the Tropics \& Subtropics, 2, 161-167.

[7] Krupinsky, J.M. and Tanaka, D.L. (2001) Leaf Spot Diseases on Winter Wheat Influenced by Nitrogen, Tillage, and Haying after a Grass-Alfalfa Mixture in the Conservation Reserve Program. Plant Disease, 85, 785-789. https://doi.org/10.1094/PDIS.2001.85.7.785

[8] Magnen, H. (2008) Balanced Crop Nutrition: Fertilizing for Crop and Food Quality. Turkish Journal of Agriculture and Forestry, 3, 183-193.

[9] Gothoskar, S.S., Scheffer, R.P., Walker, J.C. and Stahmann, M.A. (1995) The Role of Enzymes in the Development of Fusarium Wilt of Tomato. Indian Phytopathology, 3, 381-387.

[10] Sharma, P. (2007) Vegetables Diseases, Diagnosis and Biomanagement. Aavishkar Publishers, Distributors, Jaipur India, 218.

[11] Matho, J. and Schure, P.S.J. (2001) "Kersek", a Bacterial Disease of Rice. Contribution, General Agriculture Research Bogor, 117, 1-17.

[12] Ruckstuhl, M. (1998) Population Structure and Epidemic of Bipolaris sorakiniana in Rice-Wheat Cropping Pattern in Nepal. In: Duveiller, E., Dubin, H.J., Reeves, J. and Mchab, A., Eds., Helminthosporium Blights of Wheat Spot Blotch and Tan Spot, CIMMYT, Veracruz, $1328 \mathrm{p}$.

[13] Dissayanke, N. and Wickramsinghe, W. (2009) Effect of Nitrogen, Phosphorus and 
K Application on the Occurrence and Severity of Narrow Brown Leaf Spot in Different Rice Varieties. Journal of Plant Protection Sciences, 1, 51-54.

[14] Bockus, W.W. and Davis, M.A. (2003) Effect of Nitrogen Fertilizers on Severity of Tan Spot of Winter Wheat. Plant Disease, 77, 508-510.

https://doi.org/10.1094/PD-77-0508

[15] Chaurasia, P.C.P. and Duveiller, E. (2006) Management of Leaf Blight (Bipolaris sorokiniana) Disease of Wheat with Cultural Practices. Nepal Agriculture Research Journal, 7, 63-69.

[16] Harris, G. (1997) Potassium Deficiency in Cotton Linked to Leaf Spot Disease. Better Crops, 2, 10-12.

[17] Jayasena, K. and Brennan, R. (2007) Potassium Deficient Soil Is More Susceptible to Powdery Mildew Diseases. Department of Agriculture and Food, Western Australia Farmnote 216.

[18] Prabhu, A.S., Barbosa, M.P. and Filipp, M.C. (1999) Relationship between Potassium Fertilization and Panicle Blast Severity in Upland Rice. Pesquisa Agropecuária Brasileira, 9, 1729-1732.

[19] Tajani, M., Douira, A., Haloui, N.E. and Benkirane, R. (1993) Impact of Fertilization on Disease Development and Yield Components. Cahiers Options Mediterraneennes, 3, 95-99.

[20] William, J. and Smith, S.G. (2001) Correcting Potassium Deficiency Can Reduce Rice Stem Diseases. Better Crops, 85, 7-9.

[21] Smith, B.J. (2009) Influence of Nitrogen, Phosphorus, and Potassium on the Severity of Strawberry Anthracnose Crown rot. Acta Horticulturae, 842, 235-238. https://doi.org/10.17660/ActaHortic.2009.842.37

[22] IRRI (International Rice Research Institute) (2004) Rice, Almanac. 3rd Edition, International Rice Research Institute, Los Baños, 59-235.

[23] Steel, R., Torrie, J. and Dickey, D. (1997) Principles and Procedures of Statistics. A Biometrical Approach. 3rd Edition, McGraw Hill Book Co., New York.

Submit or recommend next manuscript to SCIRP and we will provide best service for you:

Accepting pre-submission inquiries through Email, Facebook, LinkedIn, Twitter, etc. A wide selection of journals (inclusive of 9 subjects, more than 200 journals)

Providing 24-hour high-quality service

User-friendly online submission system

Fair and swift peer-review system

Efficient typesetting and proofreading procedure

Display of the result of downloads and visits, as well as the number of cited articles

Maximum dissemination of your research work

Submit your manuscript at: http://papersubmission.scirp.org/

Or contact as@scirp.org 